\title{
Practical Stability of Control Processes Governed by Semiexplicit DAEs
}

\author{
R. Juárez, V. Azhmyakov, and A. Poznyak \\ Departamento de Control Automático, CINVESTAV, Avenida Instituto Politécnico Nacional 2508, \\ 07300 México, DF, Mexico \\ Correspondence should be addressed to R. Juárez; rjuarez@ctrl.cinvestav.mx
}

Received 29 October 2012; Revised 20 February 2013; Accepted 10 March 2013

Academic Editor: Jitao Sun

Copyright (c) 2013 R. Juárez et al. This is an open access article distributed under the Creative Commons Attribution License, which permits unrestricted use, distribution, and reproduction in any medium, provided the original work is properly cited.

This paper deals with a new approach to robust control design for a class of nonlinearly affine control systems. The dynamical models under consideration are described by a special class of structured implicit differential equations called semi-explicit differentialalgebraic equations (of index one), in the presence of additive bounded uncertainties. The proposed robust feedback design procedure is based on an extended version of the classical invariant ellipsoid technique that we call the Attractive Ellipsoid (AE) method. The theoretic schemes elaborated in our contribution are illustrated by a simple computational example.

\section{Introduction}

Recently the interest the new powerful robust control design approaches to the nonconventional control systems has been significantly increased (see, e.g., [1-10]). For example, the robust stabilizing of dynamic models described by implicit differential equations has emerged as a challenging problem of the modern systems theory [11-13]. In our paper, we consider a particular family of control processes governed by so-called semi-explicit differential algebraic equations (DAEs) of index one in the presence of bounded uncertainties. We restrict our consideration to the mathematical models of nonlinear systems with affine structures. Our aim is to elaborate a constructive control design scheme that guarantees the practical stability (stabilization with respect to a bounded "small" region) of the closed-loop system realizations. The concrete robust control synthesis approach proposed in our contribution is a consequence of the newly established "Attractive Ellipsoid" (AE) method established in $[5,6,8,10,14-16]$.

In our paper, we investigate a particular family of dynamic processes characterized by the implicit differential equations with the so called quasi-Lipschitz right-hand sides that can be reduced to the semi-explicit DAEs of index one (see, e.g., [12] for details). The main mathematical tool used in our paper is an appropriate extension of the classic Lyapunovbased techniques (consult $[1,3,4,9,17])$. However, we apply these techniques in combination with the descriptor method [18]. The effectiveness of the method we propose is illustrated by means of an example of a strictly nonlinear implicit control system.

The remainder of our paper is organized as follows. Section 2 contains the problem formulation and related concepts. The basic analytic results related to the AE method are collected in Section 3. In this part of the paper we also discuss a robust feedback control design procedure based on a "minimal-size" attractive ellipsoid. Section 4 is devoted to the corresponding computational technique that extends the elaborated methodology. In fact, we reduce the robust controller design problems to an auxiliary LMI-constrained optimization problem. Section 5 summarizes the paper.

\section{Problem Formulation and Some Preliminaries}

Let us consider the following initial value problem for the nonlinear implicit system with an affine structure

$$
\begin{gathered}
E \dot{x}(t)=f(x(t))+B u(x(t))+\eta(t), \quad t \in \mathbb{R}_{+}, \\
x(0)=x_{0},
\end{gathered}
$$




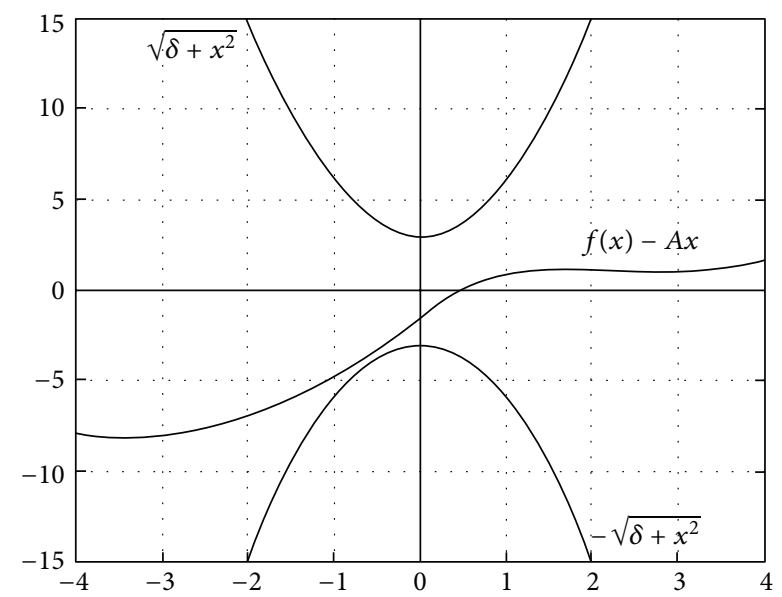

Figure 1: The one-dimensional domain of quasi-Lipschitz functions.

where $E \in \mathbb{R}^{n \times n}$ is a given matrix with $\operatorname{det}\{E\}=0$ and $B \in \mathbb{R}^{n \times m}$. By $x(t) \in \mathbb{R}^{n}, t \in \mathbb{R}_{+}$and $x \in \mathbb{R}^{n}, u(x) \in$ $\mathbb{R}^{m}$, we denote here the $n$-dimensional state vector and the $m$-dimensional feedback-type control vector, respectively. Problem (1) constitutes an adequate modeling framework for a wide class of control systems characterized by an implicit state equation in presence of some bounded uncertainties $\eta(t) \in \mathbb{R}^{n}, t \in \mathbb{R}_{+}$, where $\|\eta(t)\|_{\mathrm{Q}_{\eta}} \leq 1$ (see [19]). By $\|\cdot\|_{H}$, where $H$ is a $n \times n$-dimensional symmetric positive defined matrix, we denote a weighted Euclidean norm $\|x\|_{H}:=$ $\sqrt{x^{T} H x}$. Let us now make the basic technical assumptions associated with (1):

(i) $f: \mathbb{R}^{n} \rightarrow \mathbb{R}^{n}$ is a continuously differentiable function with a bounded derivative and moreover,

$$
\|f(x)-A x\|_{Q_{f}}^{2} \leq \delta+\|x\|_{Q_{x}}^{2} \quad \forall x \in \mathbb{R}^{n}, h \in \mathbb{R}_{+} ;
$$

(ii) $\eta(\cdot)$ is assumed to be a bounded measurable function;

(iii) $u(x)$ is chosen in the form of a linear feedback, namely, $u(x)=K x$, where $K \in \mathbb{R}^{m \times n}$.

Here, $Q_{f}$ and $Q_{x}$ are symmetric positive defined matrices. Through this paper, functions $f(\cdot)$ that satisfy the first basic hypothesis are called quasi-Lipschitz functions (the first hypothesis from the above main conditions). The simple onedimensional illustration of the quasi-Lipschitz restrictions is given on Figure 1.

We now use the basic assumption and rewrite (1) in the following equivalent form

$$
\begin{gathered}
E \dot{x}(t)=A x(t)+(f(x(t))-A x(t)+B K x(t)+\eta(t)), \\
x(0)=x_{0}, \quad t \in \mathbb{R}_{+} .
\end{gathered}
$$

Let us introduce some useful concepts and facts associated with a matrix pair $\left(\Gamma_{1}, \Gamma_{2}\right)$, where $\Gamma_{1}, \Gamma_{2} \in \mathbb{R}^{n \times n}$. We refer to [12] for the additional technical details.
Definition 1. A matrix pair $\left(\Gamma_{1}, \Gamma_{2}\right)$ is called regular if the characteristic polynomial $p(\lambda):=\operatorname{det}\left(\lambda \Gamma_{1}-\Gamma_{2}\right), \lambda \in \mathbb{R}$ is not a zero polynomial. A matrix pair which is not regular is called singular.

Definition 2. Two pairs of matrices $\left(\Gamma_{1}, \Gamma_{2}\right)$ and $\left(\Sigma_{1}, \Sigma_{2}\right)$, $\Sigma_{1}, \Sigma_{2} \in \mathbb{R}^{n \times n}$, are called strongly equivalent if there exist nonsingular matrices $\Theta, \Delta \in \mathbb{R}^{n \times n}$ such that

$$
\Sigma_{1}=\Theta \Gamma_{1} \Delta, \quad \Sigma_{2}=\Theta \Gamma_{2} \Delta
$$

if this is the case, we write $\left(\Gamma_{1}, \Gamma_{2}\right) \sim\left(\Sigma_{1}, \Sigma_{2}\right)$.

Recall that a matrix $N$ is called a nilpotent matrix of the index $v \in \mathbb{N}$ if $N^{v}=0$ and $N^{\nu-k} \neq 0$ for all $1 \leq k \leq v-1$, $k \in \mathbb{N}$. Using the above concepts, we are now ready to discuss so called Weierstrass canonical form (see, e.g., [12]).

Theorem 3. Let $\left(\Gamma_{1}, \Gamma_{2}\right)$ be regular. Then, one has

$$
\left(\Gamma_{1}, \Gamma_{2}\right) \sim\left(\left[\begin{array}{cc}
I & 0 \\
0 & N
\end{array}\right],\left[\begin{array}{ll}
J & 0 \\
0 & I
\end{array}\right]\right),
$$

where $J$ is a matrix in Jordan canonical form and $N$ is a nilpotent matrix also in Jordan canonical form. Moreover, it is allowed that the one or the other block is not present.

Theorem 3 gives rise to further retransformation of the original closed-loop system (3). Let us additionally assume that the given matrix pair $(E, A)$ is regular, namely,

$$
(E, A) \sim\left(\left[\begin{array}{cc}
I & 0 \\
0 & N
\end{array}\right],\left[\begin{array}{ll}
J & 0 \\
0 & I
\end{array}\right]\right),
$$

for some nonsingular matrices $\Pi$ and $\Psi$. Introduce a coordinate transformation given by $x=\Psi z$ and multiply both sides of equation in (3) by $\Pi$ (from the left); we now rewrite (3) as a system

$$
\begin{aligned}
\dot{z}_{1}(t)= & J z_{1}(t) \\
& +\Pi(f(\Psi z(t))-A \Psi z(t)+B K \Psi z(t)+\eta(t)) \\
N \dot{z}_{2}(t)= & z_{2}(t) \\
& +\Pi(f(\Psi z(t))-A \Psi z(t)+B K \Psi z(t)+\eta(t)), \\
& z(0)=\Psi^{-1} x_{0},
\end{aligned}
$$

where $z:=\left(z_{1}, z_{2}\right)^{T}$ such that $\operatorname{dim}\left\{z_{1}\right\}=\operatorname{dim}\{J\}$ and $\operatorname{dim}\left\{z_{2}\right\}=n-\operatorname{dim}\left\{z_{1}\right\}$. Assume that the nilpotent matrix $N$ in (7) has the nilpotency index $\nu$. In that case the second equation in (7) implies the following algebraic equation:

$$
\begin{aligned}
& N^{\nu-1} z_{2}(t) \\
& \quad+N^{\nu-1} \Pi(f(\Psi z(t)) \\
& \quad-A \Psi z(t)+B K \Psi z(t)+\eta(t))=0 .
\end{aligned}
$$


Finally, we obtain a system of so-called semi-explicit differential algebraic equations (DAEs)

$$
\begin{aligned}
\dot{z}_{1}(t)= & J z_{1}(t) \\
& +\Pi(f(\Psi z(t))-A \Psi z(t)+B K \Psi z(t)+\eta(t)), \\
& 0=G\left(z_{1}, z_{2}, t\right), \quad z(0)=\Psi^{-1} x_{0},
\end{aligned}
$$

where $G\left(z_{1}, z_{2}, t\right):=N^{\nu-1} z_{2}(t)+N^{\nu-1} \Pi(f(\Psi z(t))-A \Psi z(t)+$ $B K \Psi z(t)+\eta(t)),(9)$ is equivalent to the originally given initial value problem (3).

Definition 4 (see [12]). A DAE of the form

$$
\dot{x}=g_{1}(x, y, t), \quad 0=g_{2}(x, y, t)
$$

is called semi-explicit DAE. Where $x \in \mathbb{R}^{n}, y \in \mathbb{R}^{m}$ are dependent variables, $g_{1}(\cdot, \cdot, \cdot), g_{2}(\cdot, \cdot, \cdot)$ are continuously differentiable functions.

In our paper we also restrict our consideration to implicit systems (3) which can be transformed, via matrix transformation, into a semi-explicit DAE of the form (9) with differential index one.

Recall that a differentiation index of DAE (9) is a most minimal number $L \in \mathbb{N}$ such that

$$
\begin{aligned}
& \frac{d^{(l)}}{d t^{(l)}}\left(N^{\nu-1} z_{2}(t)\right. \\
& +N^{\nu-1} \Pi(f(\Psi z(t))-A \Psi z(t) \\
& \quad+B K \Psi z(t)+\eta(t)))=0,
\end{aligned}
$$

where $l=1, \ldots, L$, implies an semi-explicit DAE with respect to $z_{2}$ (see [12] for details). Equation above has the next form for index one:

$$
\begin{aligned}
\frac{d}{d t}\left(G\left(z_{1}, z_{2}, t\right)\right)= & \frac{\partial}{\partial z_{1}}\left(G\left(z_{1}, z_{2}, t\right)\right) \dot{z}_{1} \\
& +\frac{\partial}{\partial z_{2}}\left(G\left(z_{1}, z_{2}, t\right)\right) \dot{z}_{2} \\
& +\frac{\partial}{\partial t}\left(G\left(z_{1}, z_{2}, t\right)\right) .
\end{aligned}
$$

The index one property requires that $G\left(z_{1}, z_{2}, t\right)$ be solvable with respect to $z_{2}$. In other words, the differentiation index is one if by differentiation on time of the algebraic part of an implicit differential system,

$$
\begin{aligned}
\dot{z}_{1}(t)= & J z_{1}(t) \\
& +\Pi(f(\Psi z(t))-A \Psi z(t)+B K \Psi z(t)+\eta(t)) \\
0= & \frac{\partial}{\partial z_{1}}\left(G\left(z_{1}, z_{2}, t\right)\right) \dot{z}_{1}+\frac{\partial}{\partial z_{2}}\left(G\left(z_{1}, z_{2}, t\right)\right) \dot{z}_{2} \\
& +\frac{\partial}{\partial t}\left(G\left(z_{1}, z_{2}, t\right)\right)
\end{aligned}
$$

which is solvable for $\left(\dot{z}_{1}, \dot{z}_{2}\right)$ if $\operatorname{det}\left(\left(\partial / \partial z_{2}\right)\left(G\left(z_{1}, z_{2}, t\right)\right)\right) \neq 0$. The above assumptions on the semi-explicit DAE (9) of differential index one or on the initial implicit system (3) makes it possible to use all the conventional systematic concepts associated with the dynamic behavior of the implicit systems of the type (3) including all the classic Lyapunov stability and the set stability concepts.

Let us now recall these standard definitions. A set $\mathscr{D}$ in the state space of system of a dynamic system is said to be (positively) invariant if an admissible trajectory initiated in this set remains inside the set at all future time instants. Let us denote by $\Omega(z(0))$ the (positive) limit set of (9) (the set of all positive limit points). We refer to $[1,3,4]$ for further theoretical background and useful results.

Definition 5. A compact invariant set $\mathscr{D} \subset \mathbb{R}^{n}$ of the closed-loop semi-explicit system (9) of index one is called asymptotically Lyapunov stable if $\Omega(z(0)) \in \mathscr{D}$ and

(i) for all $\epsilon>0$ there exists $\delta_{1}>0$ such that $\operatorname{dist}\{z(0), \mathscr{D}\}<\delta_{1}$ implies that $\operatorname{dist}\{z(t), \mathscr{D}\}<\epsilon, t \geq 0$ (the Lyapunov stability of the set);

(ii) there exists $\delta_{2}>0$ such that $\operatorname{dist}\{z(0), \mathscr{D}\}<\delta_{2}$ implies $\lim _{t \rightarrow \infty} \operatorname{dist}\{z(t), \mathscr{D}\}=0$ (the attraction of the set).

Here $\operatorname{dist}\{z, \mathscr{D}\}:=\min _{\tilde{z} \in \mathscr{D}}\|z-\widetilde{z}\|_{\mathbb{R}^{n}}$ is the usual Euclidean distance between a point $z \in \mathbb{R}^{n}$ and $\mathscr{D}$.

Our aim is to specify an invariant set for the closed-loop semi-explicit system (9) in the form of an ellipsoid $\mathscr{E}_{P}:=\{z \in$ $\left.\mathbb{R}^{2} \mid z^{T} P z \leq 1\right\}$, where $P$ is a symmetric positive defined $n \times n$-dimensional matrix. Using the above-mentioned classic definition of an asymptotically stable set, we now introduce the useful "attractive ellipsoid" concept.

Definition 6. We call $\mathscr{E}_{P}$ an attractive ellipsoid for system (9) if it is an asymptotically stable invariant set of (9).

The chosen gain matrix $K$ for the linear feedback causes a dynamic behavior of the complete state vector $z(t)$ such that the inequality $z^{T}(t) P z(t) \leq 1$ holds. The last can be considered in an exact or any approximative sense. The resulting dynamic behavior of the closed-loop system can be interpreted as a stability in a "practical" sense.

From the point of view of an implementable control applications we are usually interested to construct an attractive ellipsoid $\mathscr{E}_{P}$ that possesses a minimal size (in an adequate sense). This requirement can be formalized in the form of a specific minimization problem subject to the characteristic parameters of $\mathscr{E}_{P}$. This minimality property can be formalized by the following minimization problem:

$$
\begin{aligned}
& \text { minimize } \operatorname{tr}\left\{P^{-1}\right\}, \\
& \text { subject to } P>0, P=P^{T}, K \in \Upsilon,
\end{aligned}
$$

where $\Upsilon \subset \mathbb{R}^{m \times n}$ is the set of matrices which ensure invariance of attractive ellipsoid $\mathscr{E}_{P}$. The basic optimization problem (14) determines a suitable control function of the linear feedback type that guarantees asymptotical stability 
and the invariance of the designed ellipsoidal region $\mathscr{E}_{P}$ (called attractive ellipsoid). Evidently, next we need to give a constructive characterization of the set $\Upsilon$ and propose an implementable method for a computational treatment of the optimization problem (14). The above-mentioned concrete specification of the set $\Upsilon$ will be proceeded (in the next sections) by means of a Lyapunov-like analysis and using the technique of the linear matrix inequalities (see [20] for LMIs).

\section{The Attractive Ellipsoid Method}

As mentioned in Introduction, the basic results from the Lyapunov set stability theory cannot be directly applied to the implicit nonlinear system (3). Moreover, the analogous theory for the general implicit systems (see, e.g., [11, 12, 21]) is quite sophisticated in the context of practical implementations. Motivated from that fact, our study of the asymptotical stability properties of $\mathscr{E}$ will be based on the following auxiliary result.

Lemma 7. Let a function $\mathscr{V}: \mathbb{R}_{+} \rightarrow \mathbb{R}_{+}$satisfy the following differential inequality: $\dot{\mathscr{V}}(t) \leq-\alpha \mathscr{V}(t)+\beta$, where $\alpha>0$ and $\beta>0$ are some constants. Then $\varlimsup_{t \uparrow \infty} \mathscr{V}(t) \leq \beta / \alpha$ and moreover

$$
\mathscr{V}(t) \leq \frac{\beta}{\alpha}+\left(\mathscr{V}(0)-\frac{\beta}{\alpha}\right) \exp (-\alpha t) \quad \forall t \geq 0 .
$$

Note that the presented Lemma 7 can be proved directly using the well-known properties of the linear systems and the Comparison Principle (see [9]). A function $\mathscr{V}$ from Lemma 7 is further called a "storage function".

Our aim is to show that under some additional assumptions the combined function $V(x(t))$, where $x(\cdot)$ is a solution to (3) for a suitable gain matrix $K \in \Upsilon$, satisfies all the conditions of the Lemma 7 . With this aim let us use the Lyapunov-like analytic tool and introduce the following "energetic" function $V: \mathbb{R}^{n} \rightarrow \mathbb{R}_{+}$associated with the original implicit system (3):

$$
V(x):=x^{T} P x, \quad P=P^{T}, P>0 .
$$

Note that the symmetric positive defined matrix $P$ here is the same matrix as in (14). Let us consider this energetic function, namely, $V(x(t))$, evaluated along the trajectories of the initial system (3). We use the additional notation $\sigma(\eta(t), x(t)):=$ $(\eta(t)+f(x(t))-A x(t))$. We are now ready to formulate our main theoretic result.

Theorem 8. Let $\alpha>0, \beta:=1+\delta>0$. Then the Lie derivative

$$
\begin{aligned}
\left.D V(x(t))\right|_{(2)}:= & \nabla V(x(t)) \\
& \times[A x(t)+(f(x(t)) \\
& \quad-A x(t)+B K x(t)+\eta(t))]
\end{aligned}
$$

of the combined function $V(x(\cdot))$ satisfies the following inequality:

$$
D V(x(t))+\alpha V(x(t))-\beta \leq Z^{T}(t) W\left(P, P_{1}, K, \alpha\right) Z(t),
$$

where $Z(t)=\left(x^{T}(t), \dot{x}^{T}(t), \sigma^{T}(\eta(t), x(t))\right)^{T}$ is the extended state vector, $P_{1} \in \mathbb{R}^{n \times n}$, and $W\left(P, P_{1}, K, \alpha\right)$ is a matrixfunction. If $W\left(P, P_{1}, K, \alpha\right)$ is chosen as a negative defined matrix, then the combined function $V(x(\cdot))$ has all the properties of a storage function $\mathscr{V}(\cdot)$.

Proof. Let $V(x)=x^{T} P x$ and $P_{1} \in \mathbb{R}^{n \times n}$. Using the idea of the "descriptor method" (see [18] for details), we can represent $D V(x(t))$ as follows:

$$
\begin{aligned}
& D V(x(t))= D V(x(t)) \\
& \\
&+2\left\langle\left[P x(t)+P \dot{x}(t)+P_{1} \sigma(\eta(t), x(t))\right],\right. \\
& \\
&(-E \dot{x}(t)+(A+B K) x(t) \\
&+\sigma(\eta(t), x(t)))\rangle,
\end{aligned}
$$

where the second addend in the last formula is an effective zero. Applying this representation and taking into consideration the next useful effective zero given by $\sigma(\eta(t), x(t))^{T} Q_{\eta} \sigma(\eta(t), x(t))-\sigma(\eta(t), x(t))^{T} Q_{\eta} \sigma(\eta(t), x(t))$, we get

$$
\begin{aligned}
D V(x(t))+\alpha V(x(t))-\beta \\
=Z^{T}(t) \bar{W}\left(P, P_{1}, K, \alpha\right) Z(t)-\beta \\
\quad+\sigma(\eta(t), x(t))^{T} Q_{\eta} \sigma(\eta(t), x(t)) \\
\quad-\sigma(\eta(t), x(t))^{T} Q_{\eta} \sigma(\eta(t), x(t)) \\
=Z^{T}(t) \widetilde{W}\left(P, P_{1}, K, \alpha\right) Z(t)-\beta \\
\quad+\sigma(\eta(t), x(t))^{T} Q_{\eta} \sigma(\eta(t), x(t)) .
\end{aligned}
$$

Here we defined

$$
\widetilde{W}:=\left(\begin{array}{lll}
\widetilde{W}_{11} & \widetilde{W}_{12} & \widetilde{W}_{13} \\
\widetilde{W}_{21} & \widetilde{W}_{22} & \widetilde{W}_{23} \\
\widetilde{W}_{31} & \widetilde{W}_{32} & \widetilde{W}_{33}
\end{array}\right)
$$

with

$$
\begin{gathered}
\widetilde{W}_{11}:=P A+A^{T} P+P B K+K^{T} B^{T} P+\alpha P, \\
\widetilde{W}_{12}:=P-P E+A^{T} P+K^{T} B^{T} P, \\
\widetilde{W}_{13}:=P+A^{T} P_{1}+K^{T} B^{T} P_{1}, \\
\widetilde{W}_{21}:=P-E^{T} P+P A+P B K, \\
\widetilde{W}_{22}:=-\left(P E+E^{T} P\right), \quad \widetilde{W}_{23}:=P-E^{T} P_{1}, \\
\widetilde{W}_{31}:=P+P_{1}^{T} A+P_{1}^{T} B K, \\
\widetilde{W}_{32}:=P-P_{1}^{T} E, \quad \widetilde{W}_{33}:=P_{1}^{T}-Q_{\eta},
\end{gathered}
$$

where $\bar{W}\left(P, P_{1}, K, \alpha\right)$ is the same matrix-function $\widetilde{W}\left(P, P_{1}\right.$, $K, \alpha$ ) replacing $\widetilde{W}_{11}$ by

$$
\bar{W}_{33}:=P_{1}^{T} .
$$


Using the boundedness conditions from Section 2, we easily obtain $\|f(x)-A x\|_{\mathrm{Q}_{\eta}}^{2} \leq \delta+h\|x\|^{2},\|\eta\|_{\mathrm{Q}_{\eta}}^{2} \leq 1$. We now put $\beta=1+\delta$ and derive the final estimation

$$
\begin{aligned}
D V( & x(t))+\alpha V(x(t))-\beta \\
= & Z^{T}(t) \widetilde{W}\left(P, P_{1}, K, \alpha\right) Z(t)-\beta \\
& +\|\sigma(\eta(t), x(t))\|_{Q_{\eta}}^{2} \\
= & Z^{T}(t) \widetilde{W}\left(P, P_{1}, K, \alpha\right) Z(t)-\beta \\
& +\|\eta(t)+f(x(t))-A x(t)\|_{Q_{\eta}}^{2} \\
\leq & Z^{T}(t) \widetilde{W}\left(P, P_{1}, K, \alpha\right) Z(t)-\beta \\
& +\|\eta(t)\|_{Q_{\eta}}^{2}+\|f(x(t))-A x(t)\|_{Q_{\eta}}^{2} \\
\leq & Z^{T}(t) \widetilde{W}\left(P, P_{1}, K, \alpha\right) Z(t)-\beta+1 \\
& +\delta+h\|x(t)\|^{2} \\
\leq & Z^{T}(t) W\left(P, P_{1}, K, \alpha\right) Z(t)-\beta+1+\delta \\
\leq & Z^{T}(t) W\left(P, P_{1}, K, \alpha\right) Z(t),
\end{aligned}
$$

where term $h\|x(t)\|^{2}$ is included into the new matrix function $W\left(P, P_{1}, K, \alpha\right)$ and $\beta=1+h . W\left(P, P_{1}, K, \alpha\right)$ is the same matrix-function $\widetilde{W}\left(P, P_{1}, K, \alpha\right)$ replacing $\widetilde{W}_{11}$ by

$$
W_{11}:=P A+A^{T} P+P B K+K^{T} B^{T} P+(\alpha+h) P .
$$

Evidently, the negative definiteness of $W\left(P, P_{1}, K, \alpha\right)$ guarantees the properties of the storage function $\mathscr{V}(\cdot)$ (see Lemma 7 ) for the combined function $V(x(\cdot))$.

Using Theorem 8, we are now able to characterize the abstract constraint set $\Upsilon$ in the main optimization problem (14) by a constructive way. Recall that $\Upsilon \subset \mathbb{R}^{m \times n}$ is the set of the admissible gain matrices that guarantee the attractiveness of the ellipsoid $\mathscr{E}_{P}$ (see Definition 6). Therefore, the main problem (14) has now the following form:

$$
\begin{aligned}
& \text { minimize } \operatorname{tr}\left\{P^{-1}\right\} \\
& \text { subject to } P>0, P=P^{T}, \\
& W\left(P, P_{1}, K, \alpha\right)<0
\end{aligned}
$$

with a symmetric block-matrix $W\left(P, P_{1}, K, \alpha\right)$. Let us now note that due to the bilinear structure of $W\left(P, P_{1}, K, \alpha\right)$ the negative definiteness of this matrix-function constitutes a numerically sophisticated question. The nonlinear nature of the obtained multidimensional inequality in (26) evidently involves a possible relaxation approach. We next replace the above bilinear matrix inequality by an auxiliary system of linear matrix inequalities (see [20] for LMIs). This expected relaxed system will provide a basis for the further numerical treatment of the main optimization problem (26). Introduce the following symmetric matrix-function:

$$
W_{r}\left(P, P_{1}, Y, \alpha\right):=M^{T} W\left(P, P_{1}, K, \alpha\right) M
$$

where $M:=\operatorname{diag}\left(P^{-1}, P^{-1}, P_{1}^{-1}\right) \in \mathbb{R}^{3 n \times 3 n}$ is an auxiliary matrix. The component wise definition of $W_{r}\left(P, P_{1}, Y, \alpha\right)$ can be given by the corresponding expressions:

$$
\begin{gathered}
W_{r_{11}}=A P^{-1}+P^{-1} A^{T}+B Y+Y^{T} B^{T}+(\alpha+h) P^{-1}, \\
W_{r_{12}}=P^{-1}-E P^{-1}+P^{-1} A^{T}+Y^{T} B^{T}, \\
W_{r_{13}}=P_{1}^{-1}+P^{-1} A^{T}+Y^{T} B^{T}, \\
W_{r_{21}}=P^{-1}-P^{-1} E^{T}+A P^{-1}+B Y, \\
W_{r_{22}}=-\left(E P^{-1}+P^{-1} E^{T}\right), \quad W_{r_{23}}=P_{1}^{-1}-P^{-1} E^{T}, \\
W_{r_{31}}=P_{1}^{-1}+A P^{-1}+B Y, \\
W_{r_{32}}=P_{1}^{-1}-E P^{-1}, \quad W_{r_{33}}=P_{1}^{-1}-P_{1}^{-1} Q_{\eta} P_{1}^{-1},
\end{gathered}
$$

where $Y:=K P^{-1} \in \mathbb{R}^{m \times n}$ is an artificial variable. The last expressions for the component of $W_{r}\left(P, P_{1}, K, \alpha\right)$ are linear functions (excepting the term $W_{r_{33}}$ ) with respect to the basic variables $\left(P, P_{1}, Y,\right)$ for a fixed scalar component $\alpha$. To avoid the technical problems with the bilinear structure of $W_{r_{33}}$ of the matrix $W_{r}\left(P, P_{1}, Y, \alpha\right)$ it is always be possible to ask for a $P_{1}^{-1}$ which satisfies inequality

$$
P_{1}^{-1} Q_{\eta} P_{1}^{-1}>\rho P_{1}^{-1}
$$

for some real constant $\rho>0$ and then majorize $W_{r}\left(P, P_{1}\right.$, $K, \alpha)<F_{r}\left(P, P_{1}, Y, \alpha\right)$, where $F_{r}\left(P, P_{1}, Y, \alpha\right)$ is a matrix that is equal to $W_{r}\left(P, P_{1}, Y, \alpha\right)$ with replacing of the element $W_{r_{33}}$ by the overestimated term $F_{r_{33}}:=(1-\rho) P_{1}^{-1}$. We can apply the celebrated Schur technique to inequality above to obtain

$$
\chi:=\left(\begin{array}{cc}
Q_{\eta} & I_{n} \\
I_{n} & \rho^{-1} P_{1}^{-1}
\end{array}\right)>0,
$$

where $I_{n}$ is the identity matrix of the size $n \times n$ and $\rho>0$ is a suitable constant. Finally, in position of the original bilinear inequality in (26) we obtain the following matrix inequality that has a linear structure under assumption of fixed scalar parameter $\alpha$ :

$$
\mathscr{W}:=\operatorname{diag}\left(F_{r}\left(P, P_{1}, Y, \alpha\right),-\chi\right)<0,
$$

where $\chi$ is defined above. Note that all terms in $\mathscr{W}$ are linear. Therefore, the main optimization problem (14) has now the following relaxed form:

$$
\begin{aligned}
& \text { minimize } \operatorname{tr}\left\{P^{-1}\right\} \\
& \text { subject to } P>0, P=P^{T}, \\
& \mathscr{W}\left(P, P_{1}, Y, \alpha\right)<0
\end{aligned}
$$

with a symmetric block-matrix $\mathscr{W}\left(P, P_{1}, Y, \alpha\right)$. 
Theorem 9. Assume that the relaxed optimization problem (32) has an optimal solution $\left(P^{o p t}, P_{1}^{o p t}, Y^{o p t}\right)$ for a fixed scalar variable $\alpha$. Then the following 5-tuple $\left(P^{o p t}, P_{1}^{o p t}, K^{o p t}, \alpha\right)$ is also an optimal solution to the initial nonlinearly constrained problem (26) with $K^{o p t}:=Y^{o p t} P^{o p t}$.

The last result is a direct consequence of the one-toone transformations (introduced above) $K=Y P$ for some admissible $P$ and $Y$. Therefore, the formal relaxation of the initial problem does not really extend the solutions set of the original problem (26). The obtained equivalent optimization problem (32) with linear type restrictions (LMIs) provides an analytic basis for the implementable numerical algorithms associated with the given problem (14) initially formulated in the abstract form. Recall that (14) determines the practically stable linear feedback control strategy for the implicit dynamic system (3). This control design is based on the minimal size attractive ellipsoid $\mathscr{E}_{P}$ and in fact defined by the selection of the optimal gain matrix $K^{\text {opt }}$.

\section{Numerical Aspects of the Attractive Ellipsoid Method}

In this part of the paper we apply the theoretic results from Section 3 to the practical stability analysis of implicit systems (3). Our aim is to propose a realizable attractive ellipsoid based numerical scheme for the computational robust control design. Let us first summarize the constructive approaches discussed above in the form of a Conceptual Algorithm.

\section{Algorithm 10.}

(0) Put $l=0$, select an initial positive scalar $\alpha^{(0)}$, and choose an accuracy $\epsilon>0$.

(1) Solve the optimization problem (32).

(2) Use the obtained solution $\Phi^{(l)}:=\left(P^{(l)}, P_{1}^{(l)}, Y^{(l)}\right)$ to (32) and solve the system of linear inequalities (with respect to the unknown pair $\alpha$ )

$$
\begin{gathered}
\mathscr{W}\left(P^{(l)}, P_{1}^{(l)}, Y^{(l)}, \alpha\right)<0 \\
\alpha>0 .
\end{gathered}
$$

(3) Get a solution $\alpha^{(l)}$ to (33). Increase $l=l+1$ and solve (32). If $\left|P^{(l)}-P^{(l-1)}\right|<\epsilon$, go to Step (4). Else, go to Step (2).

(4) Stop. Determine $\left(P^{\mathrm{opt}}, P_{1}^{\mathrm{opt}}, Y^{\mathrm{opt}}, \alpha^{\mathrm{opt}}\right):=\left(P^{(l)}, P_{1}^{(l)}\right.$, $\left.Y^{(l)}, \alpha^{(l)}\right)$ and $K^{\text {opt }}:=Y^{(l)} P^{(l)}$.

The minimization problem (32) in Steps (1) and (3) constitutes an important part of the proposed algorithm. It can be solved using some standard numerical techniques. The same is also true with respect to the auxiliary subproblem (33). Note that (32) (under assumption of the fixed scalar parameter $\alpha$ ) is a linear optimization problem with respect to the unknown matrices. Let us illustrate the effectiveness of the proposed Conceptual Algorithm 10 by way of an academic example.

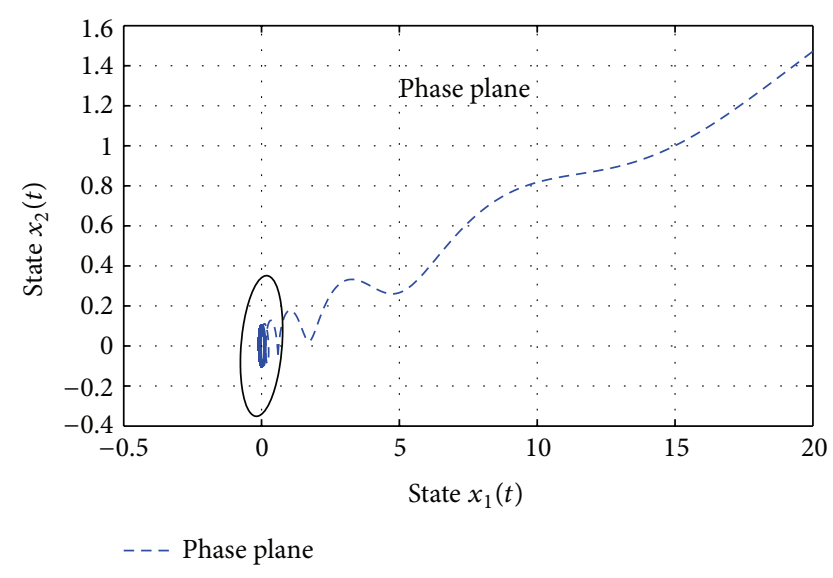

FIGURE 2: Phase plane of nonlinear system.

Example 11. Consider the semi-explicit DAE system of the type (1) with index one and concrete dates

$$
\begin{aligned}
& E=\left(\begin{array}{ll}
1 & 0 \\
0 & 0
\end{array}\right), \quad f(x)=\left(-0.1 \operatorname{sign}\left(x_{1}\right), x_{2}\right), \\
& A=\left(\begin{array}{cc}
-0.1 & 0 \\
0 & 1
\end{array}\right), \quad B=\left(\begin{array}{l}
1 \\
1
\end{array}\right) .
\end{aligned}
$$

We evidently have the following simple estimations that corresponds to our main assumptions (see Section 2)

$$
\|f(x)-A x\|^{2}=\left|-0.1\left(\operatorname{sign}\left(x_{1}\right)-x_{1}\right)\right|^{2} \leq(0.1)^{2}+(0.1)^{2} x_{1}^{2} .
$$

We now fix $h=\delta=0.01$ and the initial value $\alpha^{(0)}=10^{-5}$. Using the Conceptual Algorithm discussed above, we next obtain

$$
\begin{aligned}
P^{\mathrm{opt}} & =\left(\begin{array}{ll}
0.05879 & 0.00645 \\
0.00645 & 0.01235
\end{array}\right), \\
K^{\mathrm{opt}} & =\left(\begin{array}{ll}
-0.056 & -0.032
\end{array}\right) .
\end{aligned}
$$

Using the obtained value $K^{\mathrm{opt}}$, and applying the standard MATLAB toolboxes Ode23t, Ode15s, and Ode15i, we are able to calculate the trajectory $x(\cdot)$ of the closed-loop system (3). We have considered the following initial conditions $x_{0}=$ $(20,1.157)^{T}, \dot{x}_{0}=(-1.257,10)^{T}$. The external perturbation is selected as $\eta(t)=1 \times 10^{-3}(\sin t, \sin t)^{T}$. The application of Conceptual Algorithm 10 in the given system induces the following numerical results indicated on Figures 2 and 3. Figure 2 represents the state coordinates of the closed-loop system (3). The calculated optimal matrix $P^{\text {opt }}$ determines the corresponding attractive ellipsoid of the minimal size that contains the origin. Figure 3 shows the time evolution of the same state components $\left(x_{1}(t), x_{2}(t)\right)$.

Finally note that the applied theoretic method and the corresponding computational results constitute a robust behavior of the resulting system in the sense of the effective disturbances rejection. 


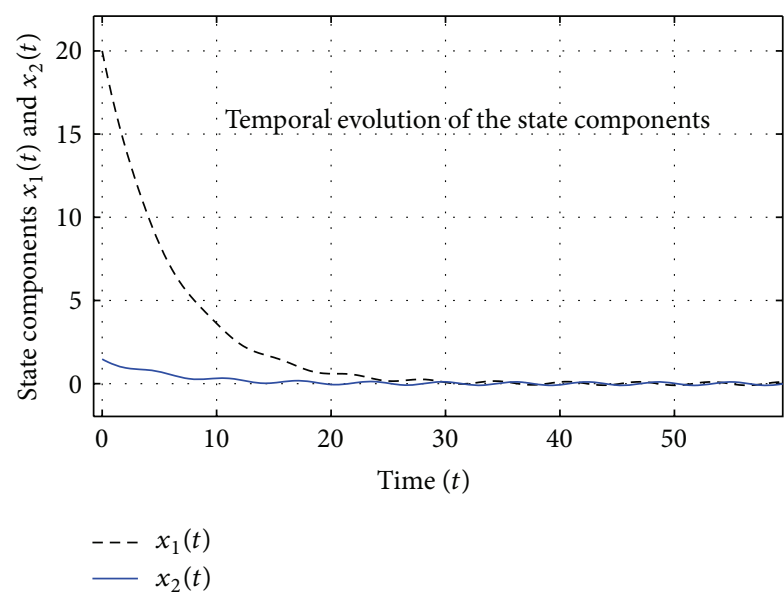

Figure 3: Temporal evolution of trajectories $x_{1}$ and $x_{2}$.

\section{Concluding Remarks}

In this paper we developed a new analytic and computational method for the robust feedback control design of nonlinear implicit dynamic systems governed by semi-explicit DAEs of index one and closed by a linear control law. The proposed design procedure in our contribution constitutes an extension of the conventional invariant ellipsoid technique (called the attractive ellipsoid method). From the analytic point of view, the elaborated methodology generates an admissible linear feedback control strategy that guarantee existence and a concrete characterization of a minimal size invariant ellipsoid associated with the system realization. The obtained ellipsoidal invariant set is asymptotically stable in the sense of Lyapunov. Finally note that the main analytic idea and the associated numerical treatment can be easily applied to some alternative linear and nonlinear control processes that incorporate some natural bounded uncertainties.

\section{References}

[1] F. Blanchini and S. Miani, Set-Theoretic Methods in Control, Systems \& Control: Foundations \& Applications, Birkhäuser Boston Publishers, Boston, Mass, USA, 2008.

[2] M. A. Dahleh and J. B. Pearson, Jr., "Optimal rejection of persistent disturbances, robust stability, and mixed sensitivity minimization," IEEE Transactions on Automatic Control, vol. 33, no. 8, pp. 722-731, 1988.

[3] W. M. Haddad and V. Chellaboina, Nonlinear Dynamical Systems and Control, Princeton University Press, Princeton, NJ, USA, 2008.

[4] H. K. Khalil, Nonlinear Systems, Prentice Hall, Upper Saddle River, NJ, USA, 2002.

[5] A. B. Kurzhanski and P. Varaiya, "Ellipsoidal techniques for reachability under state constraints," SIAM Journal on Control and Optimization, vol. 45, no. 4, pp. 1369-1394, 2006.

[6] M. Mera, A. Poznyak, and V. Azhmyakov, "On the robust control design for a class of continuous-time dynamical systems with a sample-data output," in Proceedings of the 18th IFAC World Congress, pp. 5819-5824, Milano, Ialy, 2011.
[7] A. N. Michel, L. Hou, and D. Liu, Stability of Dynamical Systems: Continuous, Discontinuous, and Discrete Systems, Systems \& Control: Foundations \& Applications, Birkhäuser Boston Publishers, Boston, Mass, USA, 2008.

[8] B. T. Polyak and M. V. Topunov, "Suppression of bounded exogeneous disturbances: output control," Automation and Remote Control, vol. 69, pp. 801-818, 2008.

[9] A. S. Poznyak, Advanced Mathematical Tools for Automatic Control Engineers: Deterministic Techniques, vol. 1, Elsevier B. V., Amsterdam, The Netherlands, 2008.

[10] A. Poznyak, V. Azhmyakov, and M. Mera, "Practical output feedback stabilisation for a class of continuous-time dynamic systems under sample-data outputs," International Journal of Control, vol. 84, no. 8, pp. 1408-1416, 2011.

[11] L. Dai, Singular Control Systems, vol. 118 of Lecture Notes in Control and Information Sciences, Springer, Berlin, Germany, 1989.

[12] P. Kunkel and V. Mehrmann, Differential-Algebraic Equations: Analysis and Numerical Solution, EMS Textbooks in Mathematics, EMS Publishing, Zürich, Switzerland, 2006.

[13] K. Takaba, "Stability analysis of interconnected implicit systems based on passivity," in Proceedings of the 15th Triennial World Congress, Barcelona, Spain, 2002.

[14] V. Azhmyakov, "On the geometric aspects of the invariant ellipsoid method: application to the robust control design," in Proceedings of the 50th Conference on Decision and Control, pp. 1353-1356, Orlando, Fla, USA, 2011.

[15] S. A. Nazin, B. T. Polyak, and M. V. Topunov, "Rejection of bounded exogenous disturbances by the method of invariant ellipsoids," Automation and Remote Control, vol. 68, no. 3, pp. 467-486, 2007.

[16] A. Polyakov and A. Poznyak, "Lyapunov function design for finite-time convergence analysis: "Twisting" controller for second-order sliding mode realization," Automatica, vol. 45, no. 2, pp. 444-448, 2009.

[17] E. D. Yakubovich, "Solution of a problem in the optimal control of a discrete linear system," Automation and Remote Control, vol. 36, pp. 1447-1453, 1976.

[18] E. Fridman, "Descriptor discretized Lyapunov functional method: analysis and design," IEEE Transactions on Automatic Control, vol. 51, no. 5, pp. 890-897, 2006.

[19] J. D. Glover and F. C. Schweppe, "Control of linear dynamic systems with set constrained disturbances," IEEE Transactions on Automatic Control, vol. 16, pp. 411-423, 1971.

[20] S. Boyd, L. El Ghaoui, E. Feron, and V. Balakrishnan, Linear Matrix Inequalities in System and Control Theory, vol. 15 of SIAM Studies in Applied Mathematics, SIAM, Philadelphia, Pa, USA, 1994.

[21] W. C. Rheinboldt, "On the existence and uniqueness of solutions of nonlinear semi-implicit differential-algebraic equations," Nonlinear Analysis, vol. 16, no. 7-8, pp. 647-661, 1991. 


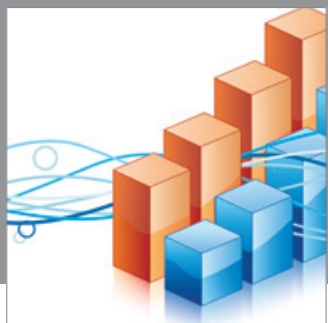

Advances in

Operations Research

mansans

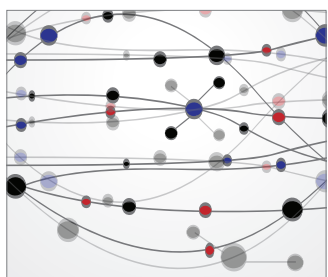

The Scientific World Journal
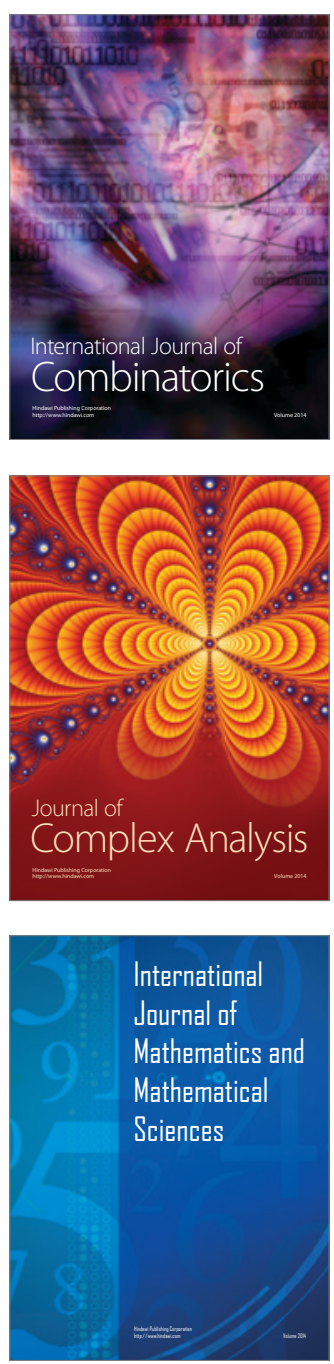
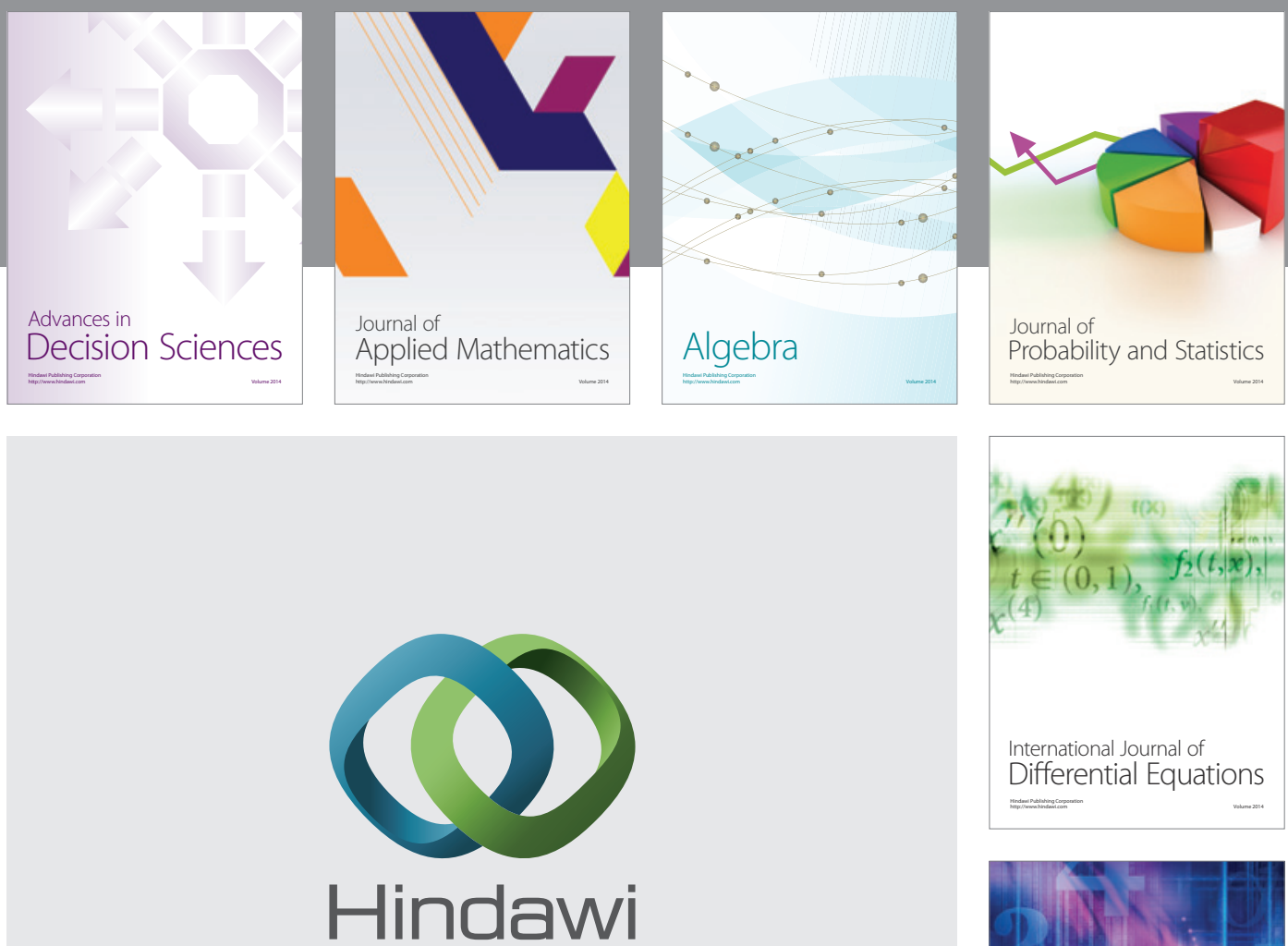

Submit your manuscripts at http://www.hindawi.com
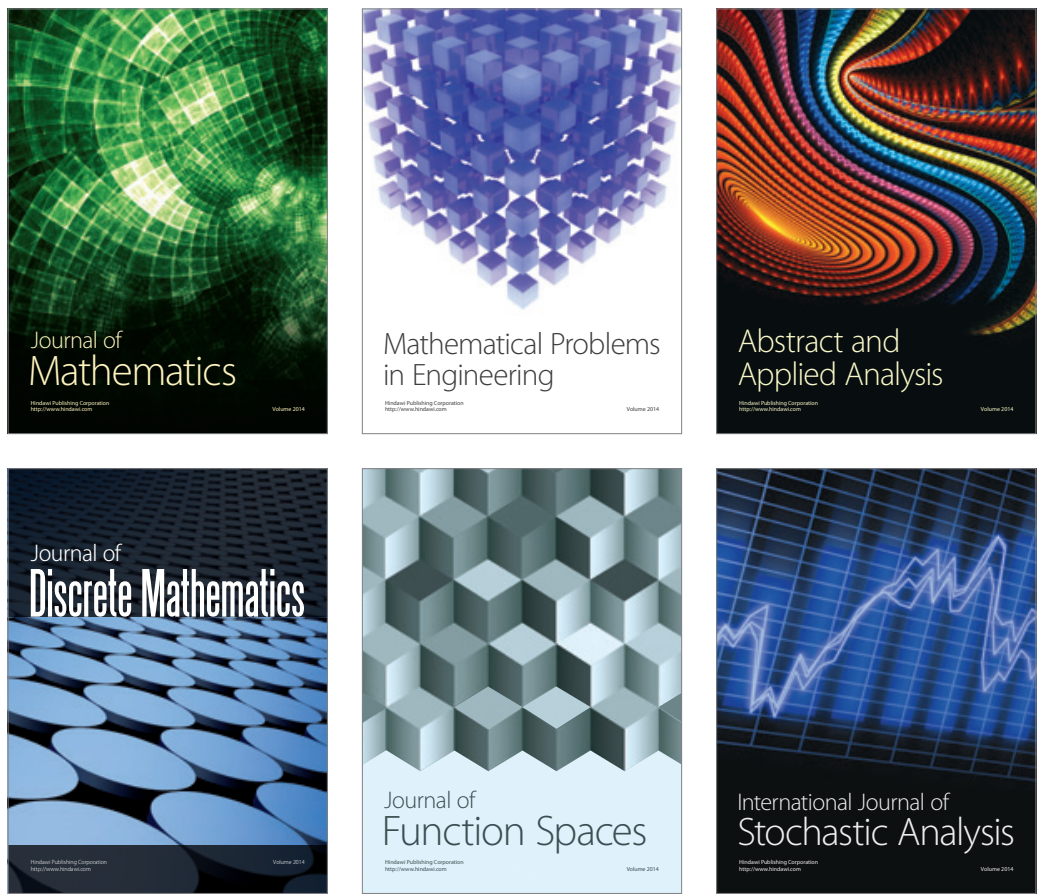

Journal of

Function Spaces

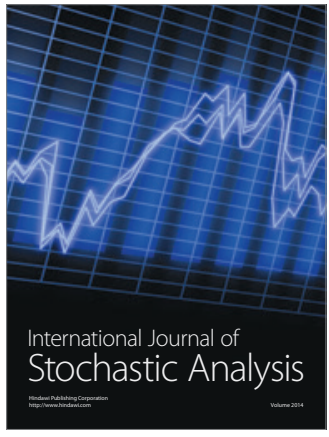

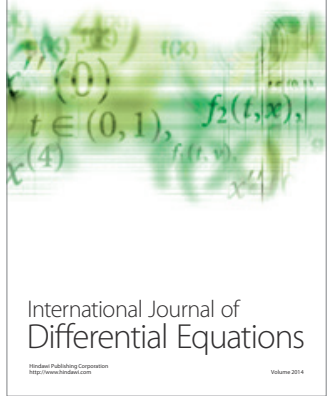
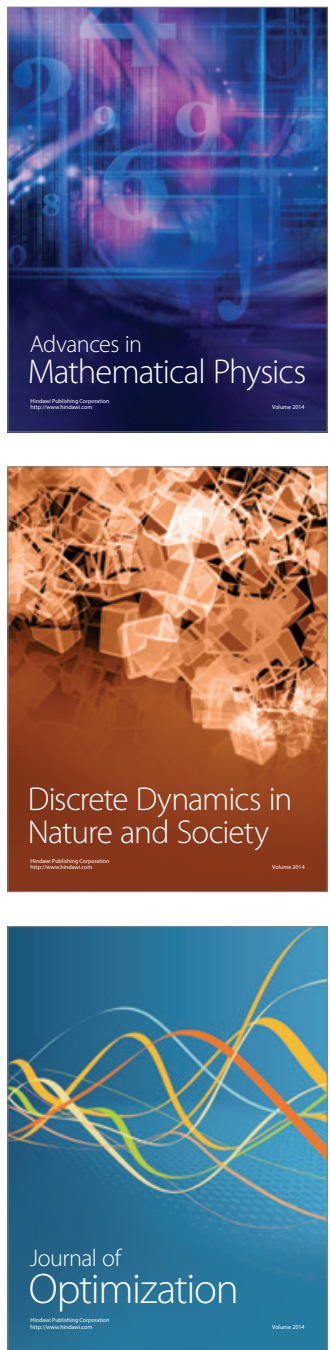